$P P B=$

\title{
Nota Científica \\ Crescimento de ipê-amarelo na região central do Rio Grande do Sul
}

Anna Paula Lora Zimmermann'1, Daniele Guarienti Rorato ${ }^{1}$, Thomas Schroder ${ }^{1}$, Paulo Renato Schneider ${ }^{1}$, Adriana Falcão Dutra ${ }^{1}$

${ }^{1}$ Universidade Federal de Santa Maria, Centro de Ciências Rurais, Av. Roraima, 1.000, Bairro Camobi, CEP 95105-900, Santa Maria, RS, Brasil

"Autor correspondente:

zimmermann-a@hotmail.com

Termos para indexação:

Handroanthus chrysotrichus

Modelo de crescimento

Plantação

Index terms:

Handroanthus chrysotrichus

Growth model

Plantation

Histórico do artigo:

Recebido em 03/06/2013

Aprovado em 28/11/2014

Publicado em 31/12/2014

doi: 10.4336/2014.pfb.34.80.546

\begin{abstract}
Resumo - Objetivou-se analisar o crescimento em diâmetro e altura de indivíduos de Handroanthus chrysotrichus através de modelos em função da idade, em um povoamento no Município de São Pedro do Sul, RS. Os dados são de três árvores representativas da população e foram obtidos através de análise de tronco. O crescimento em diâmetro dos indivíduos de Handroanthus chrysotrichus em função da idade foi melhor explicado pelo modelo alométrico enquanto para a altura o modelo exponencial negativo apresentou melhores parâmetros estatísticos. Os modelos selecionados explicam mais de $92 \%$ da variação e se configuram como ferramentas adequadas para o manejo desta espécie.
\end{abstract}

\section{Growth of yellow ipe in central region of Rio Grande do Sul State, Brazil}

\begin{abstract}
This study aimed to analyze the growth in diameter and height of individuals Handroanthus chrysotrichus through models based on age, in a stand in the municipality of São Pedro do Sul, State of Rio Grande do Sul, Brazil. Data are from three representative trees of the population and were obtained from stem analysis. The diameter growth of Handroanthus chrysotrichus trees in a function of age was best explained by the allometric model. For height the negative exponential model showed better statistical parameters. Selected models explain more than $92 \%$ of the variation and configure as adequate tools for the management of this species.
\end{abstract}

O ipê-amarelo (Handroanthus chrysotrichus (Mart. ex A.DC.) Mattos) pertence à família Bignoniaceae, com importante relevância devido às suas inúmeras utilidades econômicas (Catão et al., 2011), ornamentais (Santana et al., 2005) e ecológicas (Souza et al, 2005). Sua madeira é pesada, resistente ao apodrecimento e ataque de cupins, possibilitando seu uso inclusive para fins nobres (Souza et al., 2005). Em geral, os indivíduos desta espécie tendem a crescer de forma reta e sem bifurcações quando plantados em reflorestamento, aumentando o índice de aproveitamento da madeira (Pinto \& Rodigheri, 2001).

Nas últimas décadas é possível notar uma maior preocupação no entendimento de como as variáveis de crescimento de espécies florestais nativas comportamse, e como é possível potencializá-las (Schneider et al., 2000; Tonini et al., 2003). O aprimoramento das técnicas de manejo e a necessidade cada vez maior de informações a respeito do crescimento dos povoamentos fez com que o uso de modelos de crescimento se tornasse uma ferramenta importante na predição do estoque de madeira (Schneider et al., 2000). Assim, este trabalho se justifica por buscar entender o comportamento do crescimento de ipêamarelo em função da idade.

O povoamento situa-se no Município de São Pedro do Sul, RS; e foi implantado com a finalidade única de 
arborização. O plantio foi em linha, em espaçamento de $3 \mathrm{~m} \mathrm{x} 2 \mathrm{~m}$ (1.666 plantas ha-1). O clima na região é subtropical Cfa, com temperatura média de $18{ }^{\circ} \mathrm{C}$ e precipitação anual de $1.700 \mathrm{~mm}$, distribuída durante todos os meses do ano (Moreno, 1961).

Para analisar o crescimento em diâmetro, volume total, incremento corrente anual em diâmetro e fator de forma, foram obtidos dados de três árvores representativas do povoamento. Tal escolha foi baseada nos diâmetros de Hohenadl, dados em função da média dos diâmetros e do desvio padrão (Machado et al., 2003), e calculados a partir do banco de dados do inventário florestal realizado na área (Rorato et al., 2008).

Os indivíduos tiveram a altura total e o diâmetro à altura do peito medidos com trena e suta, respectivamente. Foram obtidos discos nas alturas: $0,10 \mathrm{~m}, 0,70 \mathrm{~m}, 1,30$ $\mathrm{m}$, e a partir do dap de um em um metro até o ponto da altura comercial. As fatias foram identificadas e polidas gradativamente, com lixa nas gramaturas entre $60 \mathrm{e}$ 220 , com objetivo de tornar os anéis mais visíveis e a superfície da fatia lisa, facilitando a contagem e medição dos anéis de crescimento. A dimensão acumulada dos anéis de crescimento foi realizada sobre os raios traçados a partir da medula, utilizando aparelho óptico LINTAB II, com deslocamento horizontal e precisão de $0,01 \mathrm{~mm}$. Os dados foram analisados pelo procedimento proreg, procglm e procnlin no pacote estatístico SAS (Statistical Analysis System) versão 9.1.

A árvore 3, por apresentar maior idade, também apresentou maiores valores para todas as variáveis apresentadas, diminuindo de acordo com a idade para as árvores 1 e 2 (Tabela 1).
Tabela 1. Variáveis dendrométricas encontradas para Ipêamarelo, na Região Central do RS.

\begin{tabular}{cccccc}
\hline Árvore & Idade & Dap $\mathbf{( c m})$ & $\mathbf{H t}(\mathbf{m})$ & $\mathbf{g ~ ( \mathbf { m } ^ { 2 } )}$ & $\mathbf{V ~ ( \mathbf { m } ^ { 3 } )}$ \\
\hline 1 & 10 & 4,6 & 7,6 & 0,0017 & 0,0072 \\
2 & 6 & 3,0 & 6,2 & 0,0007 & 0,0022 \\
3 & 12 & 6,4 & 6,9 & 0,0032 & 0,0099 \\
\hline
\end{tabular}

Onde: Dap = diâmetro a $1,30 \mathrm{~m}$ do solo; $\mathrm{Ht}=$ altura total; $\mathrm{g}=$ área basal; $\mathrm{V}=$ volume

Para a análise do crescimento em diâmetro no dap e altura total foram testados os modelos alométricos $\left(\mathrm{y}=\mathrm{b}_{0} * \mathrm{t}^{\mathrm{b} 1}\right)$, exponencial modificado $\left(\mathrm{y}=\mathrm{b}_{0} * \exp \left(\mathrm{b}_{1} / \mathrm{t}\right)\right) \mathrm{e}$ $\log$ aritmo $\left(\mathrm{y}=\mathrm{b}_{0}+\mathrm{b}_{1} * \ln \mathrm{t}\right)$.

Os dados utilizados para este estudo permitiram ajustar modelos de crescimento em diâmetro e altura para a espécie analisada (Tabela 2).

De uma forma geral, os resultados mostram que as equações de diâmetro e altura explicam de forma satisfatória os dados, visto que estas apresentaram altos valores de coeficiente de determinação, com valores superiores a 0,90 e 0,73, para diâmetro e altura, respectivamente. $\mathrm{O}$ erro padrão da estimativa também apresentou valores baixos, satisfatórios para as equações testadas, demonstrando que há pouca dispersão dos dados ao longo da linha de regressão.

Para a variável dap, a equação 1 estimou melhor os dados pelo fato de apresentar maior coeficiente de determinação e menor erro padrão da estimativa, obtendo o menor valor ponderado. No entanto, para a variável altura total estes requisitos foram preenchidos pelo modelo 2, o qual apresentou melhor ajuste para esta variável conforme os mesmos critérios de seleção utilizados para o diâmetro.

Tabela 2. Resultado dos parâmetros estatísticos das equações testadas para estimar o crescimento em diâmetro e altura de Ipê-amarelo, na região central do Rio Grande do Sul.

\begin{tabular}{llccccc}
\hline \multirow{2}{*}{ Equação } & \multirow{2}{*}{ Variável } & \multicolumn{2}{c}{ Coeficientes } & \multirow{2}{*}{$\mathbf{R}^{\mathbf{2}}$} & \multirow{2}{*}{ Sxy } \\
\cline { 3 - 4 } & & $\mathbf{b}_{\mathbf{0}}$ & $\mathbf{b}_{\mathbf{1}}$ & & \\
\hline \multirow{2}{*}{ Alométrica (1) } & Dap & 0,3723 & 1,1449 & 0,9640 & 0,3016 \\
& Altura total & 1,7394 & 0,5862 & 0,7338 & 0,8083 \\
\cline { 1 - 4 } Exponencial modificado (2) & Dap & 10,9226 & $-7,5468$ & 0,9272 & 0,4288 \\
& Altura total & 9,5209 & $-3,6107$ & 0,7735 & 0,7456 \\
\cline { 1 - 2 } & Dap & $-2,8659$ & 3,4103 & 0,9082 & 0,4815 \\
& Altura total & $-0,4100$ & 3,0838 & 0,7523 & 0,7609 \\
\hline
\end{tabular}

Onde: Dap é o diâmetro a $1,30 \mathrm{~m}$ do solo; $\mathrm{b}_{0}$ e $\mathrm{b}_{1}=$ parâmetros da equação; $\mathrm{R}^{2}=$ coeficiente de determinação; Sxy $=$ erro padrão da estimativa. 
Na Figura 1 são apresentadas as tendências de crescimento em diâmetro e altura das árvores analisadas e a reta de crescimento média ajustada para os dados,

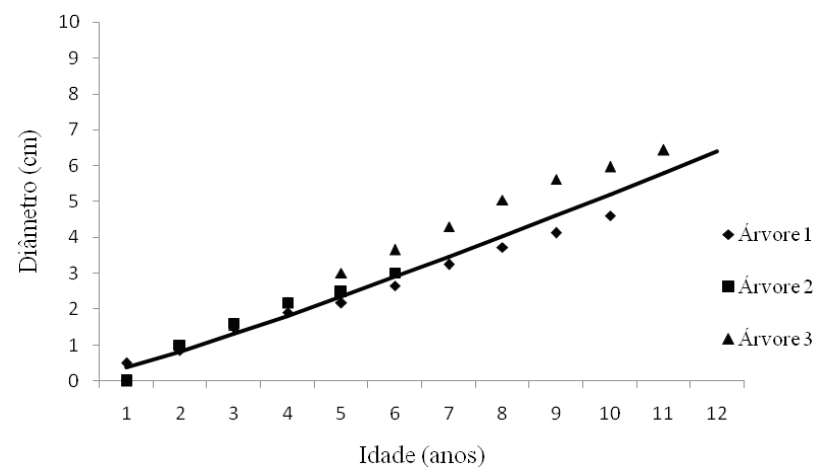

Figura 1. Tendência do crescimento em diâmetro e altura das árvores de ipê-amarelo analisadas na região central do Rio Grande do Sul.

O modelo escolhido para estimar a variável diâmetro apresentou melhor ajuste nas menores idades. A partir do quinto ano, aproximadamente, a reta obtida pelo modelo acabou se tornando um valor médio estimado. O mesmo fato também pode ser observado para a altura. É possível notar que as árvores apresentam grande incremento em altura nos primeiros dez anos. A partir desta idade, os indivíduos tendem a diminuir seu crescimento em altura, como pode ser observado pelo fato da curva estar próxima ao seu ponto de estabilização. $\mathrm{O}$ mesmo não ocorre para o dap, visto que a curva de crescimento para esta variável se mantém ascendente em todas as idades analisadas (Weiskittel et al., 2011).

Finger (1992) comenta que ao se observar a dinâmica do crescimento em diâmetro e altura ao longo do tempo as diferenças na estabilização destas variáveis podem ser notadas claramente, visto que a diferença na curva da relação hipsométrica vai diminuindo com o aumento da idade, refletida pela estabilização do crescimento que ocorre na altura antes do que no diâmetro. Para escolha dos processos estatísticos envolvidos na análise de dados é necessário o conhecimento sobre a estrutura dos mesmos (Gelman, 2007). Dados longitudinais como os oriundos de análises de tronco ou de parcelas permanentes, devem ser analisados com processos estatísticos que levem em consideração a estrutura de medidas repetidas no mesmo indivíduo.

A fim de analisar se as árvores de ipê-amarelo crescem de maneira diferente em diâmetro e altura em função da idade, realizou-se a análise de covariância para os dados longitudinais. Primeiro verificou-se se existia diferença de nível entre as tendências de crescimento. obtida através da escolha do modelo que melhor descreveu os dados.

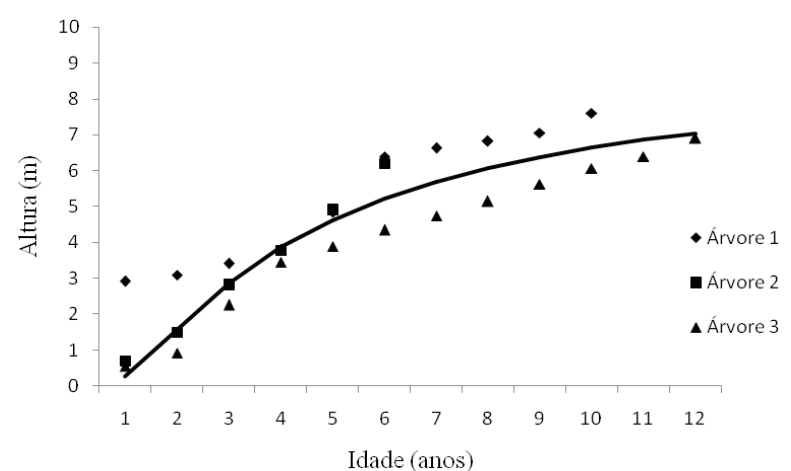

Caso a diferença fosse significativa, acrescentava-se ao modelo a variação de inclinação entre as árvores, sendo verificada novamente sua significância. Posteriormente, foram utilizadas variáveis dummy para separar as árvores em grupos diferentes entre si (Tabela 3).

Tabela 3. Análise de covariância para nível e inclinação realizada nos indivíduos analisados de ipê-amarelo na região central do Rio Grande do Sul.

\begin{tabular}{lcccc}
\hline \multicolumn{1}{c}{ FV } & GL & QM & Valor de F & Pr $>$ F \\
\hline LNT & 1 & 1,995 & 672,89 & $<0,01$ \\
ARV & 2 & 0,075 & 25,45 & $<0,01$ \\
LNT*ARV & 2 & 0,074 & 24,90 & $<0,01$ \\
\hline Parâmetro & Estimativa & Erro padrão & Valor de t & $\operatorname{Pr}>|\mathrm{t}|$ \\
\hline Intercepto & $-1,4188$ & 0.0788 & $-18,01$ & $<, 0001$ \\
LNT & 1,3612 & 0,0398 & 34,17 & $<, 0001$ \\
ARV 1 & 0,7270 & 0,1206 & 6,03 & $<, 0001$ \\
ARV 2 & 0,9513 & 0,1758 & 5,41 & $<, 0001$ \\
ARV 3 & - & - & - & - \\
LNT*ARV 1 & $-0,4057$ & 0,0636 & $-6,38$ & $<, 0001$ \\
LNT*ARV 2 & $-0,4894$ & 0,1125 & $-4,35$ & 0,0005 \\
LNT*ARV 3 & - & - & - & - \\
\hline
\end{tabular}

Onde: $\mathrm{FV}=$ fator de variação; $\mathrm{GL}=$ graus de liberdade; $\mathrm{QM}=$ quadrado médio do erro; $\mathrm{t}=$ Iidade; $\ln =$ logaritmo neperiano; Arv = árvore individual

Verifica-se que pelo menos duas árvores se diferenciam entre si quanto à inclinação e/ou quanto ao nível do modelo. No entanto, a análise por variáveis dummy demonstrou que a árvore 3 diferencia-se das outras, enquanto a árvore 1 e 2 formam um grupo homogêneo (Tabela 4). 
Tabela 4. Análise da covariância para o modelo de crescimento em altura de ipê-amarelo.

\begin{tabular}{ccccc}
\hline FV & GL & QM & Valor de $\mathbf{F}$ & Pr $>\mathbf{F}$ \\
\hline $1 / \mathrm{t}$ & 1 & 1,882 & 231,13 & $<0,01$ \\
Arv & 2 & 0,156 & 19,15 & $<0,01$ \\
\hline
\end{tabular}

Onde: $\mathrm{FV}=$ fator de variação; $\mathrm{GL}=$ graus de liberdade; $\mathrm{QM}=$ quadrado médio do erro; $\mathrm{t}$ = Idade; Arv = árvore individual.
A inclusão de variáveis dummy aos modelos de predição de crescimento aumentou consideravelmente os valores de coeficiente de determinação e reduziu os erros associados aos modelos (Tabela 5). A maior diferença ocorreu na estimativa da altura, na qual houve um aumento em $16 \%$ na precisão da estimativa do crescimento em altura com a divisão das árvores em diferentes grupos.

Tabela 5. Coeficientes do modelo gerado através da análise de covariância para o crescimento de Ipê-amarelo.

\begin{tabular}{|c|c|c|c|c|c|c|}
\hline \multirow{2}{*}{ Variável } & \multicolumn{4}{|c|}{ Coeficientes } & \multirow{2}{*}{$\mathbf{R}^{2}$} & \multirow{2}{*}{ Sxy } \\
\hline & $\mathbf{b}_{0}$ & $b_{1}$ & $\mathrm{~d}_{3}$ & $b_{0} * d_{3}$ & & \\
\hline Diâmetro a altura do peito & $-0,551$ & 0,892 & $-0,867$ & 0,469 & 0,9868 & 0,0622 \\
\hline Altura total & 2,432 & $-4,046$ & $-0,244$ & - & 0,9335 & 0,0893 \\
\hline
\end{tabular}

O gráfico que expressa os valores observados e estimados de diâmetro em função do tempo (Figura 2), mostra que a árvore 3 apresentou crescimento em diâmetro menor que as outras árvores nos primeiros três anos. No entanto, após este período seu crescimento foi maior, apresentando um diâmetro maior após os oito anos

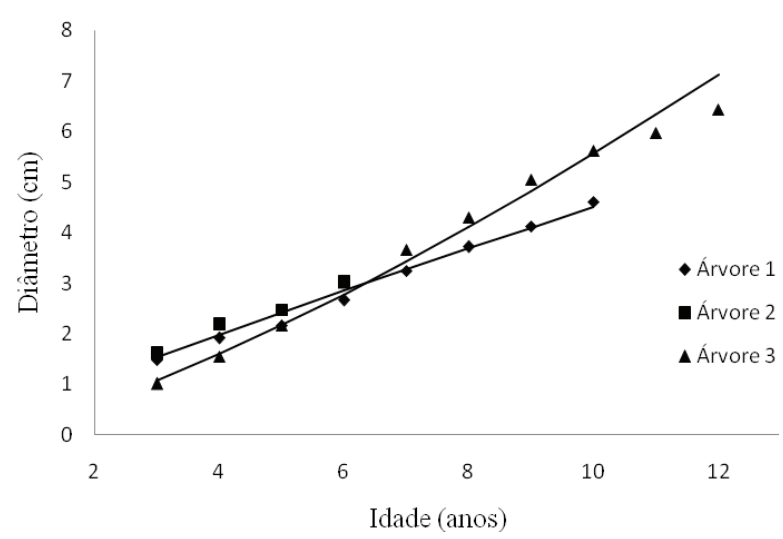

de idade, devido à liberação de indivíduos na área. Este processo é característico de árvores que foram liberadas devido à mortalidade de competidores (Fraver \& White, 2005). Ao mesmo tempo, as árvores 1 e 2 apresentam crescimento constante e não sofreram nenhum processo aparente de mudança de nível de exposição à competição.

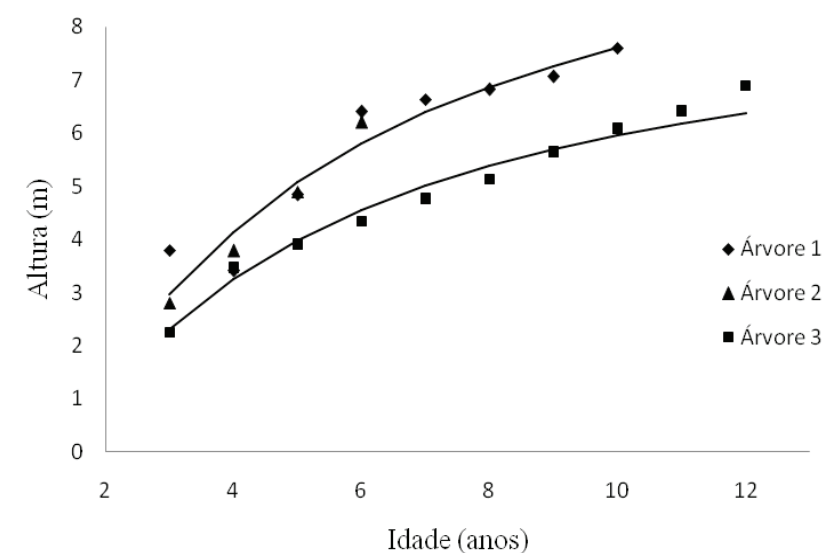

Figura 2. Valores reais e estimados para o crescimento em diâmetro e altura de ipê-amarelo obtidos através do modelo gerado pela análise de covariância.

O alto coeficiente de determinação do modelo de crescimento em altura demonstra a qualidade das estimativas geradas por este. Em um trabalho realizado com a espécie do mesmo gênero botânico, Handroanthus heptaphyllus, os valores do coeficiente de determinação para a estimativa do diâmetro à altura do peito foram semelhantes aos valores encontrados no presente trabalho (Schneider et al., 2000). Outros trabalhos que também utilizaram análise de tronco produziram resultados semelhantes tanto para espécies nativas (Milani et al., 2013) como para espécies exóticas (Elesbão \& Schneider, 2011).

Os resultados da análise de covariância demonstraram que os indivíduos analisados crescem de maneira diferente em altura e diâmetro. A análise de covariância, levando em consideração a estrutura longitudinal dos dados, melhorou consideravelmente o ajuste dos modelos. Os modelos construídos permitem estimar a altura e diâmetro de ipê-amarelo com precisão de mais de $90 \%$, sendo uma ferramenta fundamental para o manejo 
desta espécie. Assim como este, trabalhos utilizando reduzido número de árvores como os de Milani (2010) e Schneider et al. (2000) mostraram resultado satisfatórios na predição do crescimento das espécies analisadas, através da análise de tronco.

\section{Referências}

CATÃO, C.; PAES, J. B.; GOMES, J. P.; ARAÚJO, G. T. Qualidade da madeira de cinco espécies florestais para o envelhecimento da cachaça. Revista Brasileira de Engenharia Agrícola e Ambiental, Campina Grande, v. 15, n. 7, p. 741-747, 2011.

ELESBÃO, L.; SCHNEIDER, P. R. Produção do Pinus taeda L. em povoamento desbastado na região dos Campos de Cima da Serra, Rio Grande do Sul. Ciência Florestal, Santa Maria, RS, v. 21, n.1, p. 119-124, 2011.

FINGER, C. A. G. Fundamentos de biometria florestal. Santa Maria, RS: UFSM/CEPEF/FATEC, 1992. 269 p.

FRAVER, S.; WHITE, A. S. Identifying growth releases in dendrochronological studies of forest disturbance. Canadian Journal of Forest Research, Ottawa, v. 35, n. 7, p. 1648-1656, 2005.

GELMAN, A. Data analysis using regression and multilevel: hierarchical models. New York: Cambridge University Press, 2007.

MACHADO, S. A.; FIGUEIREDO FILHO, A. Dendrometria. Curitiba, 2003. 309 p.

MILANI, E. Crescimento de Podocarpus lambertii Klotzsch ex Endl. em duas regiões fitogeográficas no estado do Rio Grande do Sul. 2010. 155 f. Tese (Doutorado em Ciências Florestais) Universidade Federal de Santa Maria, RS.

MILANI, E.; SCHNEIDER, P.; CUNHA, T. da. Crescimento em diâmetro de árvores de Podocarpus lambertii em duas regiões fitogeográficas no Estado do Rio Grande do Sul, Brasil. Ciência Florestal, Santa Maria, RS, v. 23, n. 2, p. 443-448, 2013.
MORENO, J. A. Clima do Rio Grande do Sul. Porto Alegre: Secretaria da Agricultura, Diretoria de Terras e Colonização, 1961. $42 \mathrm{p}$.

PINTO, A. F.; RODIGHERI, H. R. Reflorestamento misto de espécies florestais nativas a pleno sol na região do Norte Pioneiro do Estado do Paraná. Colombo: Embrapa Florestas, 2001. 2 p. (Embrapa Florestas. Comunicado técnico, 56).

RORATO, D. G.; LENCINA, K. H.; SCHNEIDER, P. R. Abundância da regeneração natural de Tabebuia chrysotricha na depressão central do Rio Grande do Sul. In: SIMPÓSIO LATINO AMERICANO SOBRE MANEJO FLORESTAL 4., 2008, Santa Maria, RS. Produção de bens materiais e imateriais: anais. Santa Maria, RS: UFSM, 2008.

SANTANA, D. de Q.; AUER, C. G.; ROCHA, A. L. J. L.; MARQUES, E. Insetos associados ao enrolamento foliar de Tabebuia spp. em viveiros e na arborização urbana de Curitiba. Boletim de Pesquisa Florestal, Colombo, v. 50, p. 117-126, jan./jun. 2005.

SCHNEIDER, P. R.; BRENA, D. A.; FINGER, C. A. G. Manual para coleta de informações dendrométricas. Santa Maria, RS: UFSM/CEPEF/FATEC, 1988. 28 p.

SCHNEIDER, P. S. P.; SCHNEIDER, P. R.; FINGER, C. Crescimento do ipê-roxo, Tabebuia impetiginosa Martius ex AP de Candolle, na Depressão Central do Estado do Rio Grande do Sul. Ciência Florestal, Santa Maria, RS, v. 10, n. 2, p. 91-100, 2000.

SOUZA, V. C.; ANDRADE, L. A.; BRUNO, R. L. A.; CUNHA, A. O.; SOUZA, A. P. Produção de mudas de Ipê-amarelo em diferentes substratos e tamanhos de recipientes. Agropecuária Técnica, v. 26, n. 2 , p. $98-108,2005$.

TONINI, H.; FINGER, C. A. G.; SCHNEIDER, P. R. O crescimento de Nectandra megapotamica Mez., em floresta nativa na Depressão Central do Estado do Rio Grande do Sul. Ciência Rural, Santa Maria, RS, v. 33, n. 1, p. 85-90, jan/fev. 2003.

WEISKITTEL, A. R.; HANN, D. W.; KERSHAW JUNIOR, J. A.; VANCLAY, J. K. Forest growth and yield modeling. New York: John Wiley \& Sons, 2011. 